\title{
Weed Management Strategies in SRI Cultivation and Their Impact on Water Uptake by Weeds
}

\author{
D. Pal*, C. Majumder, R. K. Ghosh, D. Nongmaithem, S. Bera and S. Das
}

Dept. of Agronomy, Faculty of Agriculture, Bidhan Chandra Krishi Viswavidyalaya, Mohanpur, Nadia, W.B. (741 252), India

Article History

Manuscript No. AR1664a

Received in $25^{\text {th }}$ July, 2016

Received in revised form $27^{\text {th }}$ November, 2016

Accepted in final form $30^{\text {th }}$ November, 2016

\section{Correspondence to}

"E-mail: paldebesh@rediffmail.com

\section{Keywords}

Plant extract, SRI, water, weed management

\begin{abstract}
A field experiment with rice under SRI was conducted in kharif season of 2010 and 2011 atInstructional Farm, Jaguli, BCKV, Nadia, West Bengal to study the effect of weed management practices on water uptake by weeds in a randomized block design with 7 treatments. viz., Unweeded Control; Weed free (four hand weedings); Monocot weed treatment; Dicot weed treatment; Pretilachlor 30.7 EC @ $500 \mathrm{~g} \mathrm{ha}^{-1}$ as pre-emergence at 2 DAS followed by two wheel-hoeing at 20 and 40 DAS; Parthenium+Calotropis extract @ 5\% v/v each as pre-emergence at 2 DAS followed by two hoeing at 20 and 40 DAS; Two hand weeding at 20 and 40 DAS. All treatments resulted in significant decrease in weed population and weed biomass over unweeded control. In case of third treatment monocot weed and in case of fourth treatment dicot weed population was more than unweeded control. The consumptive use $(\mathrm{CU})$ was maximum $(364.5 \mathrm{~mm})$ in control treatment (first treatment) followed by monocot weed treatment, dicot weed treatment, hand weeding twice treatment, Parthenium+Calotropis extract@ 5\% each, Pretilachlor with two hoeing and weed free treatment. On the other hand, water use efficiency (WUE) was maximum (12.15 $\mathrm{kg} \mathrm{ha}^{-1} \mathrm{~mm}^{-1}$ ) in the weed free treatment closely followed by Pretilachlor with two hoeing and plant extract with two hoeing treatments. Highest grain yield $\left(4.39 \mathrm{t} \mathrm{ha}^{-1}\right)$ was recorded by weed free treatment. Again, Pretilachlor with two hoeing (yield 4.21 $\mathrm{t} \mathrm{ha}^{-1}$ ) and plant extract with two hoeing treatments (yield $4.07 \mathrm{t} \mathrm{ha}^{-1}$ ) are also yield promising, economic and environmentally safe.
\end{abstract}

\section{Introduction}

Rice (Oryza sativa L.) is the principle cereal crop grown under manifold agro climatic condition throughout India and is of paramount importance so far as Indian economy is concerned. In India rice is the leading food crop of the world, cultivated over an area of about 44.14 mha with a production level of $106.65 \mathrm{mt}$ and productivity of $2.416 \mathrm{tha}^{-1}$ (Anonymous, 2015). Rice plays a vital role in our food as well as nutritional security for millions of livelihood (Thorie et al., 2013). Thus the slogan Rice is life by IRRI during 2004 seems to be most appropriate (Chandrasekaran et al., 2007). Rice ranks second to wheat in terms of area harvested but in terms of importance as a food crop, rice provides more calories ha ${ }^{-1}$ than any cereal crop (De Dutta, 1981). Besides its importance as food, rice provides employment to the largest sector of the rural population in most of the Asia. With the burgeoning increase of population, demand for food is on high. It has been estimated that rice demand in 2025 will be $765 \mathrm{mt}$ in the world. As per the recent data, rice production in the World in 2014 accounts to 741.48 $\mathrm{mt}$. In respect to production, China rank first with $208.24 \mathrm{mt}$ of paddy ( $28 \%$ of global rice production), followed by India with $157.2 \mathrm{mt}$ ( $212 \%$ of global production). At the World level, average rough paddy yield is more than $4.56 \mathrm{~kg} \mathrm{ha}^{-1}$ whereas in India it is $3.58 \mathrm{t} \mathrm{ha}^{-1}$ which is nearly half of China (6.81 t ha $\left.^{-1}\right)$ (FAO Stat, 2014).

System of Rice Intensification (SRI) is worths tudying as alternative to the conventional system ofrice cultivation especially in situation of limited resource availability (Irrigation with ground water). Successful crop production under SRI method depends upon the effective weed management practice as heavy weed infestation happens in this type of establishment system. Out of several factors responsible for low yield, severe infestation of weeds in SRI cultivation is considered to be one of the major obstacles in achieving higher yield. Weeds compete with rice plants for sunlight, space, water and nutrients. The extent of yield reduction due to weeds alone is estimated to be $15-30 \%$ for transplanted rice, $30-35 \%$ for direct seeded rice under puddle condition and over $45-90 \%$ for 
upland rice culture. Here we refer to SRI as a methodology to make rice production more efficient and sustainable avoiding non judicious and lavishly utilization of water. SRI focuses on reduction in seedling age when transplanting is a familiar practice. There is an option of direct seeding, but transplanting is most common (Norman Uphoff; CIIFAD, 2010).

In recent years, farmers have inclined to chemical method of weed control due to steep rise in labour wages, traditional time consuming weed control methods and presence of morphological similar weeds in crop field. But the new and vital problems of chemical weed management like soil, water and environment pollution and herbicide resistant weeds arise. Now is the time for thinking about alternate management practices for weed problem.

On the light of above discussion, the present experiment was conducted at the Institutional Farm, Jaguli of BCKV, Nadia, West Bengal during kharif season 2010 and 2011 to find out an alternative as well as effective method of weed control and to analyze the crop-weed competition for water uptake in direct seeded kharif rice under SRI method to study the bioefficacy of chemical herbicide and botanicals for effective weed management in rice, to study the effect of weed management practices on yield of rice and develop a ecologically safe, farmers' acceptable economic method of weed control.

\section{Materials and Methods}

\subsection{Study sites}

The field experiment was conducted in SRI method of rice during kharif, 2010 and 2011 at Instructional Farm (New alluvial zone) Jaguli, BCKV, Nadia, West Bengal. The geographical location of the experimental site is situated just south of tropic of cancer with $23^{\circ} 03^{\prime} \mathrm{N}$ latitude and $88^{\circ} 53^{\prime} \mathrm{E}$ longitude with an elevation of $9.75 \mathrm{~m}$ amsl. It comes under the subtropical humid climate. The soil was alluvial with medium fertility status, having sandy clay loam in texture with slightly acidic (pH 6.21), low in organic carbon (0.65\%) having 393.54 $\mathrm{kg} \mathrm{ha}^{-1}$ nitrogen, $23.55 \mathrm{~kg} \mathrm{ha}^{-1}$ available $\mathrm{P}_{2} \mathrm{O}$ and $135.13 \mathrm{~kg} \mathrm{ha}^{-1}$ $\mathrm{K}_{2} \mathrm{O}$. The sowing of seed was done on middle of June each year in a plot size of $5 \times 4 \mathrm{~m}^{2}$.

\subsection{Treatment details}

The experiment was conducted with randomized block design consistingof seven treatments viz., $\mathrm{T}_{1}$-Unweeded control, $\mathrm{T}_{2}$ Weed free plot (all categories of weed were removed by four hand weeding at 20, 35, 50 and $65 \mathrm{DAS}$ ), $\mathrm{T}_{3}$-Monocot weed plot (Where only monocot weeds were allowed to present in rice field, other weeds were removed manually), $\mathrm{T}_{4}$-Dicot weed plot (Where only dicot weeds were allowed to present in rice field, other weeds were removed manually), $\mathrm{T}_{5}$-Pretilachlor $30.7 \mathrm{EC}$ (a) $500 \mathrm{~g} \mathrm{ha}^{-1}$ as pre-emergence at $2 \mathrm{DAS}$ followed by two times wheel hoeing at 20 and 40 DAS, $\mathrm{T}_{6}$-Parthenium + Calotropis extract @ $5 \% \mathrm{v} / \mathrm{v}$ each as pre-emergence at 2 DAS followed by two hoeing at 20 and $40 \mathrm{DAS}$ and $\mathrm{T}_{7}$-Two hand weeding at 20 and 40 DAS. Hand weeding twice was done at 20 and 40 DAS and two hoeing operations performed at 15 and 45 DAS. Herbicide as well as bio-pesticide mixture was applied as pre-emergence at 1-2 DAS. Parthenium hysterophorus and Calotropis procera are collected and leaves are crushed to make aqueous extract @ $50 \mathrm{ml} \mathrm{l}^{-1}(\mathrm{v} / \mathrm{v})$ i.e. $5 \%$ concentration of Parthenium and Calotropis each.

\subsection{Cultivation methods}

The rice variety used in this experiment was Satabdi (IET4786), which is an indica variety. The full dose of $\mathrm{P}_{2} \mathrm{O}_{5}$ and $\mathrm{K}_{2} \mathrm{O}$ (30 $\mathrm{kg} \mathrm{ha}^{-1}$ each) was applied at recommended dose as basal. Full amount of nitrogen $\left(60 \mathrm{~kg} \mathrm{ha}^{-1}\right)$ was top dressed thrice (1/3 each) at 7, 25 and 55 DAS.

\subsection{Method of data collection}

\subsubsection{Weed data}

To count the weed population and biomass in different plots, quadrate of $0.5 \times 0.5 \mathrm{~m}^{2}$ was thrown at four random places in each plot at 30 DAS and 60 DAS.

\subsubsection{Moisture percentage}

Moisture percentage was computed by oven dry method. Each time soil samples were taken from a particular soil layer of $0-15 \mathrm{~cm}$ with the help of screw auger and collected in aluminium moisture box for each layer separately. Soil samples were dried in the oven at $105^{\circ} \mathrm{C}$ for 24 hours after recording wet weight on an electric balance. Then dry weight of sample with box was recorded and moisture percentages of the samples were determined using the following formula:

$$
\text { Moisture }(\%)=\frac{\text { Wet sample weight-dry sample weight }}{\text { Dry sample weight }} \times 100
$$

\subsubsection{Consumptive use (CU)}

Consumptive use of water by rice was calculated with the following formula:

Consumptive use $(\mathrm{cm})=\sum_{\mathrm{i}=1}^{\mathrm{n}} \frac{\mathrm{M}_{1} \mathrm{i}-\mathrm{M}_{2} \mathrm{i}}{100} \times \mathrm{BDi} \times \mathrm{Di}+\mathrm{ER}$

Where,

$\mathrm{n}=$ No. of soil layers sampled in the root zone depth D.

$\mathrm{M}_{1} \mathrm{i}=$ Soil moisture percentage at the time of first sampling or after irrigation in the $\mathrm{i}^{\text {th }}$ layer.

$\mathrm{M}_{2} \mathrm{i}=$ Soil moisture percentage at the time of second sampling or before irrigation in the $i^{\text {th }}$ layer.

$\mathrm{BDi}=$ Bulk density of $\mathrm{i}^{\text {th }}$ layer $\left(\mathrm{g} \mathrm{cc}^{-1}\right)$

$\mathrm{Di}=$ Depth of $\mathrm{i}^{\text {thlayer }}$ of soil $(\mathrm{cm})$

ER=Effective rainfall $(\mathrm{cm})$ [Here, ER was calculated from the rainfall and Pan Evaporation on daily basis, the data were collected from the Department of Agricultural Meteorology 
and Physics, BCKV, Mohanpur, Nadia. The values of ER during the different crop growth stages (like 0-25 DAS, 26-50 DAS, 51-75 DAS, 76-100 DAS and 101 (DAS)-harvesting) were 4.78, 4.91, 6.84, 7.63 and $8.54 \mathrm{in} \mathrm{cm}$ and the value of ER during the all crop growing period was $32.70 \mathrm{~cm}$ ].

\subsubsection{Water use efficiency (WUE)}

Water use efficiency is defined as yield of marketable crop production per unit of water used in evaporation (ET).

Water use efficiency was determined by taking seasonal consumptive values of different treatments and yield of that treatment using the following formula:

Water use efficiency $($ WUE $)=\frac{\text { Yield }\left(\mathrm{kg} \mathrm{ha}^{-1}\right)}{\text { CU value }(\mathrm{cm})}$

It is expressed as $\mathrm{kg} \mathrm{ha}^{-1} \mathrm{~cm}^{-1}$

Soil samples collected at different times such as before sowing, at 25, 50, 75 and 100 DAS and at harvest stage from $0-15 \mathrm{~cm}$ layer for calculation of water use efficiency (WUE) of crop. Economic analysis was done to find out Benefit: Cost ratio.

\subsubsection{Statistical analysis}

The data were subjected to statistical analysis by analysis of variance method. The correlation studies were made to reveal the association among the variables in the investigation (Gomez and Gomez, 1984). As the error mean squares of the individual experiments were homogenous, combined analysis over the years were done through un-weighted analysis. Here, the interactions between years and treatments were not significant.

\section{Results and Discussion}

Predominant weeds found in the experimental plot were (Grassy weeds) Cynodon dactylon, Dactyloctenium aegyptium, Digitaria sanguinalis, Leersia hexandra, Eleusine indica, Echinochloa colonum, Echinochloa crussgalli; (Sedge weeds) Cyperus rotundus, Cyperus difformis; (Broadleaf weeds) Alternanthera phyloxeroides, Ammania baccifera, Digera arvensis, Eclipta alba, Phyllanthus niruri, Physalis minima, Scoparia dulcis, Alternanthera sessilis, Commelina benghalensis.

\subsection{Effect of treatments on weed density}

In case of weed free treatment $\left(\mathrm{T}_{2}\right)$ plot, weeds of all categories were removed at 20,35, 50 and 65 DAS manually. Treatment $\mathrm{T}_{5}$ showed (Table 1) its best performance and recorded least no. of weeds in both the dates at 30 and 60 DAS in both the years Treatment $T_{6}$ showed significantly better performance than $\mathrm{T}_{3}, \mathrm{~T}_{4}$ and $\mathrm{T}_{7}$. The botanical mixture was found effective in controlling weeds of different categories and proved as an effective and alternative method to chemical herbicide.

It is evident from the above findings that the weed free condition $\left(\mathrm{T}_{2}\right)$ was better for controlling weeds and avoiding

\begin{tabular}{|c|c|c|c|c|c|c|c|c|c|c|c|c|}
\hline \multirow[t]{2}{*}{$\begin{array}{l}\text { Treat- } \\
\text { ments }\end{array}$} & \multicolumn{2}{|c|}{ Echinochloa spp. } & \multicolumn{2}{|c|}{$\begin{array}{c}\text { Digitaria } \\
\text { sanguinalis }\end{array}$} & \multicolumn{2}{|c|}{$\begin{array}{c}\text { Leersia } \\
\text { hexandra }\end{array}$} & \multicolumn{2}{|c|}{ Other grasses } & \multicolumn{2}{|c|}{ Sedges } & \multicolumn{2}{|c|}{ Broad leaves } \\
\hline & $\begin{array}{c}30 \\
\text { DAS }\end{array}$ & $\begin{array}{c}60 \\
\text { DAS }\end{array}$ & $\begin{array}{c}30 \\
\text { DAS }\end{array}$ & $\begin{array}{c}60 \\
\text { DAS }\end{array}$ & $\begin{array}{c}30 \\
\text { DAS }\end{array}$ & $\begin{array}{c}60 \\
\text { DAS }\end{array}$ & $\begin{array}{c}30 \\
\text { DAS }\end{array}$ & $\begin{array}{c}60 \\
\text { DAS }\end{array}$ & $\begin{array}{c}30 \\
\text { DAS }\end{array}$ & $\begin{array}{c}60 \\
\text { DAS }\end{array}$ & $\begin{array}{c}30 \\
\text { DAS }\end{array}$ & $\begin{array}{c}60 \\
\text { DAS }\end{array}$ \\
\hline $\mathrm{T}_{1}$ & $\begin{array}{c}5.15 \\
*(26.00)\end{array}$ & $\begin{array}{c}5.52 \\
(30.00)\end{array}$ & $\begin{array}{c}6.57 \\
(42.67)\end{array}$ & $\begin{array}{c}7.01 \\
(48.67)\end{array}$ & $\begin{array}{c}3.53 \\
(12.00)\end{array}$ & $\begin{array}{c}3.67 \\
(13.00)\end{array}$ & $\begin{array}{c}3.72 \\
(13.33)\end{array}$ & $\begin{array}{c}3.85 \\
(14.33)\end{array}$ & $\begin{array}{c}5.64 \\
(31.33)\end{array}$ & $\begin{array}{c}6.04 \\
(36.00)\end{array}$ & $\begin{array}{c}2.25 \\
(8.67)\end{array}$ & $\begin{array}{c}3.13 \\
(9.33)\end{array}$ \\
\hline $\mathrm{T}_{2}$ & $\begin{array}{c}0.71 \\
(0.00)\end{array}$ & $\begin{array}{c}0.71 \\
(0.00)\end{array}$ & $\begin{array}{c}0.71 \\
(0.00)\end{array}$ & $\begin{array}{c}0.71 \\
(0.00)\end{array}$ & $\begin{array}{c}0.71 \\
(0.00)\end{array}$ & $\begin{array}{c}0.71 \\
(0.00)\end{array}$ & $\begin{array}{c}0.71 \\
(0.00)\end{array}$ & $\begin{array}{c}0.71 \\
(0.00)\end{array}$ & $\begin{array}{c}0.71 \\
(0.00)\end{array}$ & $\begin{array}{c}0.71 \\
(0.00)\end{array}$ & $\begin{array}{c}0.71 \\
(0.00)\end{array}$ & $\begin{array}{c}0.71 \\
(0.00)\end{array}$ \\
\hline $\mathrm{T}_{3}$ & $\begin{array}{c}5.46 \\
(29.33)\end{array}$ & $\begin{array}{c}5.61 \\
(31.00)\end{array}$ & $\begin{array}{c}6.84 \\
(46.33)\end{array}$ & $\begin{array}{c}7.18 \\
(51.00)\end{array}$ & $\begin{array}{c}3.76 \\
(13.67)\end{array}$ & $\begin{array}{c}3.89 \\
(14.67)\end{array}$ & $\begin{array}{c}3.81 \\
(14.00)\end{array}$ & $\begin{array}{c}4.14 \\
(16.67)\end{array}$ & $\begin{array}{c}5.70 \\
(32.00)\end{array}$ & $\begin{array}{c}6.12 \\
(37.00)\end{array}$ & $\begin{array}{c}0.71 \\
(0.00)\end{array}$ & $\begin{array}{c}0.71 \\
(0.00)\end{array}$ \\
\hline $\mathrm{T}_{4}$ & $\begin{array}{c}0.71 \\
(0.00)\end{array}$ & $\begin{array}{c}0.71 \\
(0.00)\end{array}$ & $\begin{array}{c}0.71 \\
(0.00)\end{array}$ & $\begin{array}{c}0.71 \\
(0.00)\end{array}$ & $\begin{array}{c}0.71 \\
(0.00)\end{array}$ & $\begin{array}{c}0.71 \\
(0.00)\end{array}$ & $\begin{array}{c}0.71 \\
(0.00)\end{array}$ & $\begin{array}{c}0.71 \\
(0.00)\end{array}$ & $\begin{array}{c}0.71 \\
(0.00)\end{array}$ & $\begin{array}{c}0.71 \\
(0.00)\end{array}$ & $\begin{array}{c}2.46 \\
(12.00)\end{array}$ & $\begin{array}{c}3.81 \\
(0.00)\end{array}$ \\
\hline $\mathrm{T}_{5}$ & $\begin{array}{c}2.73 \\
(7.00)\end{array}$ & $\begin{array}{c}2.79 \\
(7.33)\end{array}$ & $\begin{array}{c}3.13 \\
(9.33)\end{array}$ & $\begin{array}{c}3.39 \\
(11.00)\end{array}$ & $\begin{array}{c}2.04 \\
(3.67)\end{array}$ & $\begin{array}{c}2.11 \\
(4.00)\end{array}$ & $\begin{array}{c}1.95 \\
(3.33)\end{array}$ & $\begin{array}{c}2.11 \\
(4.00)\end{array}$ & $\begin{array}{c}2.54 \\
(6.00)\end{array}$ & $\begin{array}{c}3.13 \\
(9.33)\end{array}$ & $\begin{array}{c}1.66 \\
(3.33)\end{array}$ & $\begin{array}{c}1.95 \\
(3.33)\end{array}$ \\
\hline $\mathrm{T}_{6}$ & $\begin{array}{c}3.67 \\
(13.00)\end{array}$ & $\begin{array}{c}3.81 \\
(14.00)\end{array}$ & $\begin{array}{c}3.89 \\
(14.67)\end{array}$ & $\begin{array}{c}4.38 \\
(18.67)\end{array}$ & $\begin{array}{c}2.73 \\
(7.00)\end{array}$ & $\begin{array}{c}2.73 \\
(7.00)\end{array}$ & $\begin{array}{c}2.61 \\
(6.33)\end{array}$ & $\begin{array}{c}2.97 \\
(8.33)\end{array}$ & $\begin{array}{c}4.14 \\
(16.67)\end{array}$ & $\begin{array}{c}4.22 \\
(17.33)\end{array}$ & $\begin{array}{c}1.74 \\
(4.67)\end{array}$ & $\begin{array}{c}2.27 \\
(4.67)\end{array}$ \\
\hline $\mathrm{T}_{7}$ & $\begin{array}{c}4.34 \\
(18.33)\end{array}$ & $\begin{array}{c}4.37 \\
(18.67)\end{array}$ & $\begin{array}{c}4.37 \\
(18.67)\end{array}$ & $\begin{array}{c}4.85 \\
(23.00)\end{array}$ & $\begin{array}{c}3.24 \\
(10.00)\end{array}$ & $\begin{array}{c}3.19 \\
(9.67)\end{array}$ & $\begin{array}{c}3.13 \\
(9.33)\end{array}$ & $\begin{array}{c}3.49 \\
(11.67)\end{array}$ & $\begin{array}{c}4.88 \\
(23.33)\end{array}$ & $\begin{array}{c}5.02 \\
(24.67)\end{array}$ & $\begin{array}{c}1.86 \\
(6.67)\end{array}$ & $\begin{array}{c}2.54 \\
(6.00)\end{array}$ \\
\hline $\operatorname{SEm} \pm$ & 0.04 & 0.06 & 0.04 & 0.04 & 0.03 & 0.04 & 0.02 & 0.05 & 0.05 & 0.04 & 0.01 & 0.04 \\
\hline $\begin{array}{l}\mathrm{CD} \\
(p=0.05)\end{array}$ & 0.12 & 0.17 & 0.13 & 0.12 & 0.10 & 0.15 & 0.06 & 0.14 & 0.17 & 0.13 & 0.02 & 0.13 \\
\hline
\end{tabular}

*: Figures in the brackets are original values and the other figures are the square root transformation value 
crop weed competition, but the treatments $\mathrm{T}_{5}$ and $\mathrm{T}_{6}$ along with two weeding maintain their superiority over all other treatments for controlling all categories of weeds. The chemical herbicide Pretilachlor gave a good result in controlling weeds over the botanical treatment because of the fact that this treatment controlled a broad spectrum of weeds by their quick knockdown effect and also due to its inhibiting effect on cell division and cell growth of weeds. Though the botanical herbicide did not have quick knock-down effect but it is economically viable, environmentally safe, ecologically sound and socially acceptable which advocate to the sustainable agriculture and best management practice for crop cultivation. Pretilachlor gave best performance in controlling mainly grassy weeds, less of sedges and broad leaved weeds which were corroborated by Ampong and De Datta (1991); Ghosh et al. (2007).

\subsection{Effect of treatments on weed biomass}

It is evident from Table 2 that chemical herbicide in competition with mechanical control or the botanical herbicide along with the mechanical control is more promising from the economic point of view in controlling weed biomass due to their synergistic effect than singly hand weeding treatment or even weed free treatment. Treatment $\mathrm{T}_{5}$ showed (Table 2) best performance and recorded least weed biomass in both the dates at 30 and 60 DAS in both the years Treatment $T_{6}$ showed significantly better performance than $\mathrm{T}_{3}, \mathrm{~T}_{4}$ and $\mathrm{T}_{7}$. The results of this experiment are similar with the findings of Ghosh et al. (2007).

\subsection{Effect of treatments on water use and grain yield}

Monocot weeds took soil moisture $(154.7 \mathrm{~mm})$ whereas

\begin{tabular}{|c|c|c|c|c|c|c|c|c|c|c|c|c|}
\hline \multirow[t]{2}{*}{ Treatments } & \multicolumn{2}{|c|}{ Echinochloa spp. } & \multicolumn{2}{|c|}{$\begin{array}{c}\text { Digitaria } \\
\text { sanguinalis }\end{array}$} & \multicolumn{2}{|c|}{$\begin{array}{c}\text { Leersia } \\
\text { hexandra }\end{array}$} & \multicolumn{2}{|c|}{ Other grasses } & \multicolumn{2}{|c|}{ Sedges } & \multicolumn{2}{|c|}{ Broad leaves } \\
\hline & $\begin{array}{c}30 \\
\text { DAS }\end{array}$ & $\begin{array}{c}60 \\
\text { DAS }\end{array}$ & $\begin{array}{c}30 \\
\text { DAS }\end{array}$ & $\begin{array}{c}60 \\
\text { DAS }\end{array}$ & $\begin{array}{c}30 \\
\text { DAS }\end{array}$ & $\begin{array}{c}60 \\
\text { DAS }\end{array}$ & $\begin{array}{c}30 \\
\text { DAS }\end{array}$ & $\begin{array}{c}60 \\
\text { DAS }\end{array}$ & $\begin{array}{c}30 \\
\text { DAS }\end{array}$ & $\begin{array}{c}60 \\
\text { DAS }\end{array}$ & $\begin{array}{c}30 \\
\text { DAS }\end{array}$ & $\begin{array}{c}60 \\
\text { DAS }\end{array}$ \\
\hline $\mathrm{T}_{1}$ & $\begin{array}{l}{ }^{*} 1.91 \\
(3.14)\end{array}$ & $\begin{array}{c}2.02 \\
(3.57)\end{array}$ & $\begin{array}{c}2.02 \\
(3.57)\end{array}$ & $\begin{array}{c}2.21 \\
(4.37)\end{array}$ & $\begin{array}{c}1.45 \\
(1.62)\end{array}$ & $\begin{array}{c}1.51 \\
(1.77)\end{array}$ & $\begin{array}{c}1.40 \\
(1.47)\end{array}$ & $\begin{array}{c}1.51 \\
(1.80)\end{array}$ & $\begin{array}{c}2.03 \\
(3.64)\end{array}$ & $\begin{array}{c}2.07 \\
(3.79)\end{array}$ & $\begin{array}{c}2.20 \\
(4.34)\end{array}$ & $\begin{array}{c}2.25 \\
(4.56)\end{array}$ \\
\hline $\mathrm{T}_{2}$ & $\begin{array}{c}0.71 \\
(0.00)\end{array}$ & $\begin{array}{c}0.71 \\
(0.00)\end{array}$ & $\begin{array}{c}0.71 \\
(0.00)\end{array}$ & $\begin{array}{c}0.71 \\
(0.00)\end{array}$ & $\begin{array}{c}0.71 \\
(0.00)\end{array}$ & $\begin{array}{c}0.71 \\
(0.00)\end{array}$ & $\begin{array}{c}0.71 \\
(0.00)\end{array}$ & $\begin{array}{c}0.71 \\
(0.00)\end{array}$ & $\begin{array}{c}0.71 \\
(0.00)\end{array}$ & $\begin{array}{c}0.71 \\
(0.00)\end{array}$ & $\begin{array}{c}0.71 \\
(0.00)\end{array}$ & $\begin{array}{c}0.71 \\
(0.00)\end{array}$ \\
\hline $\mathrm{T}_{3}$ & $\begin{array}{c}1.95 \\
(3.30)\end{array}$ & $\begin{array}{c}2.08 \\
(3.83)\end{array}$ & $\begin{array}{c}2.16 \\
(4.16)\end{array}$ & $\begin{array}{c}2.24 \\
(4.50)\end{array}$ & $\begin{array}{c}1.52 \\
(1.81)\end{array}$ & $\begin{array}{c}1.56 \\
(1.94)\end{array}$ & $\begin{array}{c}1.44 \\
(1.58)\end{array}$ & $\begin{array}{l}1.61 \\
(2.10)\end{array}$ & $\begin{array}{c}2.04 \\
(3.68)\end{array}$ & $\begin{array}{c}2.10 \\
(3.93)\end{array}$ & $\begin{array}{c}0.71 \\
(0.00)\end{array}$ & $\begin{array}{c}0.71 \\
(0.00)\end{array}$ \\
\hline $\mathrm{T}_{4}$ & $\begin{array}{c}0.71 \\
(0.00)\end{array}$ & $\begin{array}{c}0.71 \\
(0.00)\end{array}$ & $\begin{array}{c}0.71 \\
(0.00)\end{array}$ & $\begin{array}{c}0.71 \\
(0.00)\end{array}$ & $\begin{array}{c}0.71 \\
(0.00)\end{array}$ & $\begin{array}{c}0.71 \\
(0.00)\end{array}$ & $\begin{array}{c}0.71 \\
(0.00)\end{array}$ & $\begin{array}{c}0.71 \\
(0.00)\end{array}$ & $\begin{array}{c}0.71 \\
(0.00)\end{array}$ & $\begin{array}{c}0.71 \\
(0.00)\end{array}$ & $\begin{array}{c}2.33 \\
(5.95)\end{array}$ & $\begin{array}{c}2.42 \\
(5.35)\end{array}$ \\
\hline $\mathrm{T}_{5}$ & $\begin{array}{c}1.03 \\
(0.56)\end{array}$ & $\begin{array}{c}1.12 \\
(0.75)\end{array}$ & $\begin{array}{c}1.07 \\
(0.64)\end{array}$ & $\begin{array}{c}1.29 \\
(1.17)\end{array}$ & $\begin{array}{c}0.92 \\
(0.34)\end{array}$ & $\begin{array}{c}1.03 \\
(0.56)\end{array}$ & $\begin{array}{c}0.90 \\
(0.31)\end{array}$ & $\begin{array}{c}1.02 \\
(0.55)\end{array}$ & $\begin{array}{c}1.12 \\
(0.75)\end{array}$ & $\begin{array}{c}1.25 \\
(1.07)\end{array}$ & $\begin{array}{c}1.66 \\
(2.24)\end{array}$ & $\begin{array}{c}1.76 \\
(2.61)\end{array}$ \\
\hline $\mathrm{T}_{6}$ & $\begin{array}{c}1.34 \\
(1.28)\end{array}$ & $\begin{array}{c}1.35 \\
(1.32)\end{array}$ & $\begin{array}{c}1.35 \\
(1.32)\end{array}$ & $\begin{array}{c}1.49 \\
(1.73)\end{array}$ & $\begin{array}{c}1.13 \\
(0.77)\end{array}$ & $\begin{array}{c}1.17 \\
(0.87)\end{array}$ & $\begin{array}{c}1.05 \\
(0.60)\end{array}$ & $\begin{array}{c}1.25 \\
(1.07)\end{array}$ & $\begin{array}{c}1.46 \\
(1.63)\end{array}$ & $\begin{array}{c}1.55 \\
(1.90)\end{array}$ & $\begin{array}{c}1.74 \\
(2.54)\end{array}$ & $\begin{array}{c}1.92 \\
(3.19)\end{array}$ \\
\hline $\mathrm{T}_{7}$ & $\begin{array}{c}1.48 \\
(1.70)\end{array}$ & $\begin{array}{c}1.55 \\
(1.91)\end{array}$ & $\begin{array}{c}1.48 \\
(1.68)\end{array}$ & $\begin{array}{c}1.64 \\
(2.18)\end{array}$ & $\begin{array}{c}1.24 \\
(1.03)\end{array}$ & $\begin{array}{c}1.27 \\
(1.12)\end{array}$ & $\begin{array}{c}1.15 \\
(0.83)\end{array}$ & $\begin{array}{c}1.44 \\
(1.58)\end{array}$ & $\begin{array}{c}1.79 \\
(2.70)\end{array}$ & $\begin{array}{c}1.81 \\
(2.77)\end{array}$ & $\begin{array}{c}1.86 \\
(2.97)\end{array}$ & $\begin{array}{c}2.01 \\
(3.54)\end{array}$ \\
\hline $\operatorname{SEm} \pm$ & 0.01 & 0.01 & 0.01 & 0.01 & 0.01 & 0.01 & 0.01 & 0.01 & 0.01 & 0.01 & 0.01 & 0.01 \\
\hline $\mathrm{CD}(p=0.05)$ & 0.02 & 0.03 & 0.03 & 0.03 & 0.03 & 0.04 & 0.03 & 0.03 & 0.03 & 0.03 & 0.04 & 0.02 \\
\hline
\end{tabular}

": Figures in the brackets are original values and the other figures are the square root transformation value

dicot weeds could extract $153.9 \mathrm{~mm}$ (Table 3 ). In case of $\mathrm{T}_{5}$ (Pretilachlor30.7 EC@ $500 \mathrm{~g} \mathrm{ha}^{-1}$ as pre-emergence at 2 DAS followed by two wheel-hoeing at 20 and 40 DAS), minimum soil moisture was extracted by weeds $(152.4 \mathrm{~mm})$ and was closely followed by treatment $\mathrm{T}_{6}$ (Parthenium+Calotropis extract@ $@ 5 \% \mathrm{v} / \mathrm{v}$ each as pre-emergence at 2 DAS followed by two hoeing at 20 and 40 DAS) with value $152.9 \mathrm{~mm}$.

Highest rice grain yield $\left(4.39 \mathrm{t} \mathrm{ha}^{-1}\right)$ was recorded with the weed free treatment $\left(\mathrm{T}_{2}\right)$ which is significantly higher than all other treatments. Next best treatment was $\mathrm{T}_{5}$ and $\mathrm{T}_{6}$. The mode of action of Pretilachlor is inhibition of cell division and cell

\begin{tabular}{|c|c|}
\hline Treatments & Water uptake by different types of weed $(\mathrm{mm})$ \\
\hline $\mathrm{T}_{1}$ & 155.1 \\
\hline $\mathrm{T}_{2}$ & - \\
\hline $\mathrm{T}_{3}$ & 154.7 \\
\hline $\mathrm{T}_{4}$ & 153.9 \\
\hline $\mathrm{T}_{5}$ & 152.4 \\
\hline $\mathrm{T}_{6}$ & 152.9 \\
\hline $\mathrm{T}_{7}$ & 153.4 \\
\hline
\end{tabular}


growth of weed as reported by Ampong and De Dutta (1991). This chemical showed no phytotoxic symptoms on the crop plant.

Maximum consumptive use (CU) of crop was recorded (Table 4) by $T_{1}$ (Control plot) $364.5 \mathrm{~mm}$ followed by $\mathrm{T}_{3}$ (Monocot plot), $\mathrm{T}_{4}$ (Dicot plot), where as in case of $\mathrm{T}_{6}$ (Parthenium+Calotropis extract@ $@ 5 \% \mathrm{v} / \mathrm{v}$ each as pre-emergence at $2 \mathrm{DAS}$ followed by two hoeing at 20 and $40 \mathrm{DAS}$ ) the value was $362.3 \mathrm{~mm}$, in $\mathrm{T}_{5}$ (Pretilachlor30.7 EC@ $900 \mathrm{~g} \mathrm{ha}^{-1}$ as pre-emergence at 2 DAS followed by two wheel hoeing at 20 and 40 DAS) $361.8 \mathrm{~mm}$ and $\mathrm{T}_{2}$ (Weed free) $361.2 \mathrm{~mm}$. The results of this experiment were in conformity with the findings of Farooq et al. (2011).

Highest water use efficiency also followed the similar trend set by grain yield of rice. Maximum water use efficiency of crop was recorded (Table 4$)$ by $\mathrm{T}_{2}\left(12.15 \mathrm{~kg} \mathrm{ha}^{-1} \mathrm{~mm}^{-1}\right)$ followed by $\mathrm{T}_{5}\left(11.64 \mathrm{~kg} \mathrm{ha}^{-1} \mathrm{~mm}^{-1}\right), \mathrm{T}_{6}\left(11.23 \mathrm{~kg} \mathrm{ha}^{-1} \mathrm{~mm}^{-1}\right), \mathrm{T}_{7}(10.64 \mathrm{~kg}$ $\left.\mathrm{ha}^{-1} \mathrm{~mm}^{-1}\right) \mathrm{T}_{4}\left(9.77 \mathrm{~kg} \mathrm{ha}^{-1} \mathrm{~mm}^{-1}\right), \mathrm{T}_{3}\left(9.37 \mathrm{~kg} \mathrm{ha}^{-1} \mathrm{~mm}^{-1}\right)$ and $\mathrm{T}_{1}$ $\left(8.15 \mathrm{~kg} \mathrm{ha}^{-1} \mathrm{~mm}^{-1}\right)$. The results of this experiment were similar with the findings of Sudhir Yadav et al. (2011).

Table 4. Consumptive use, yield, water use efficiency and Benefit:Cost ratio as influenced by crop-weed competition (pooled data)

\begin{tabular}{lcccccc}
\hline $\begin{array}{l}\text { Treat- } \\
\text { ments }\end{array}$ & $\begin{array}{c}\text { CU } \\
(\mathrm{mm})\end{array}$ & GY & WUE & $\begin{array}{c}\mathrm{CC} \\
\left(₹ \mathrm{ha} \mathrm{h}^{-1}\right)\end{array}$ & $\begin{array}{c}\text { NR } \\
\left(₹ \mathrm{fa}^{-1}\right)\end{array}$ & $\begin{array}{c}\text { B:C } \\
\text { ratio }\end{array}$ \\
\hline $\mathrm{T}_{1}$ & 364.5 & 2.97 & 8.15 & 25104.52 & 9905.48 & 1.39 \\
$\mathrm{~T}_{2}$ & 361.2 & 4.39 & 12.15 & 38304.52 & 13495.48 & 1.35 \\
$\mathrm{~T}_{3}$ & 364.1 & 3.41 & 9.37 & 35304.52 & 4990.48 & 1.14 \\
$\mathrm{~T}_{4}$ & 363.3 & 3.55 & 9.77 & 30504.52 & 11445.48 & 1.38 \\
$\mathrm{~T}_{5}$ & 361.8 & 4.21 & 11.64 & 32955.98 & 16884.02 & 1.51 \\
$\mathrm{~T}_{6}$ & 362.3 & 4.07 & 11.23 & 32429.52 & 15650.48 & 1.48 \\
$\mathrm{~T}_{7}$ & 362.8 & 3.86 & 10.64 & 32304.52 & 13225.48 & 1.41 \\
$\mathrm{SEm}^{2}$ & - & 0.02 & - & - & - & - \\
$\mathrm{CD}^{*}$ & - & 0.08 & - & - & - & - \\
\hline
\end{tabular}

CU: Consumptive use; GY: Grain yield ( $\left.\mathrm{t} \mathrm{ha}^{-1}\right)$; WUE: Water use efficiency $\left(\mathrm{kg} \mathrm{ha}^{-1} \mathrm{~mm}^{-1}\right)$; CC: Cost of cultivation; NR: Net returns; $\mathrm{CD}^{*}: \mathrm{CD}(p=0.05)$

\subsection{Economics}

Maximum net returns was recorded (Table 4$)$ by $\mathrm{T}_{5}(16884.02 ₹$ ha $\left.^{-1}\right)$ followed by $\mathrm{T}_{6}(15650.48$ ₹ ha-1 $), \mathrm{T}_{2}(13495.48$ ₹ ha-1 $), \mathrm{T}_{7}$ $\left(13225.48\right.$ ₹ $\left.\left.\mathrm{ha}^{-1}\right),\right) \mathrm{T}_{4}(11445.48$ ₹ ha-1 $), \mathrm{T}_{1}(9905.48$ ₹ ha-1 $)$ and $\mathrm{T}_{3}\left(4990.48 ₹ \mathrm{ha}^{-1}\right)$. Regarding benefit: cost ratio, highest value (1.51) was recorded by treatment $\mathrm{T}_{5}$ followed by $\mathrm{T}_{6}(1.48)$, $\mathrm{T}_{7}$ (1.41), $\mathrm{T}_{1}$ (1.39), $\mathrm{T}_{4}(1.38), \mathrm{T}_{2}(1.35)$ and $\mathrm{T}_{3}$ (1.14). The findings were supported by the results of Ghosh et al. (2007).

\section{Conclusion}

Pretilachlor 30.7 EC @ $500 \mathrm{~g} \mathrm{ha}^{-1}$ as pre-emergence at 2
DAS followed by two wheel hoeing at 20 and 40 DAS can be successfully recommended against traditional hand weeding. But considering the environmental pollution effect including soil in addition to above views, the safe botanical treatment Parthenium+Calotropis extract @ 5\% v/v each as pre-emergence at $2 \mathrm{DAS}$ followed by two hoeing at 20 and 40 DAS is highly advocated and appropriate for the present day agriculture. It also acts as an indirect method for weed control. It is ecologically sound, socially acceptable for sustainable agriculture and best management practice for crop cultivation.

\section{References}

Ampong, N.K., De Datta, S.K., 1991. A handbook for weed control in rice. IRRI, Manilla, Philippines, 113.

Chandrasekaran, B., Annadurai, K., Kavimani, R., 2007. A textbook of rice science. Scientific publishers (India), Jodhpur, India, 7.

De Dutta, S.K., 1981. Rice growing countries. In: Principles and Practices of rice production. Wiley-Interscience Publication, New York, 4-6.

Anonymous, 2015. Agricultural Statistics at a Glance, Directorate of Economics \& Statistics, Department of Agriculture, Cooperation \& Farmers Welfare, Ministry of Agriculture \& Farmers Welfare, Government of India, 84.

FAO Stat, 2014. Available from: http://www.fao.org/faostat/ en/\#data/QC. Accessed on December, 2016.

Farooq, M., Siddique, K.H.M., Rehman, H., Aziz, T., Lee D.J., Wahid, A., 2011. Rice direct seeding: experiences, challenges and opportunities. Soil and Tillage Research 111(2), 87-98.

Ghosh, R.K., Mondal, S.S., Maiti, S., 2007. Modern weed science manual, Department of Agronomy, BCKV, Mohanpur, West Bengal, India.

Gomez, K.A., Gomez, A.A., 1984. Statistical Procedures for Agricultural Research ( $2^{\text {nd }}$ Edn). Singapore. John Wiley $\&$ Sons.

Norman, U., 2010. Responses to Frequently-asked questions about the system of rice intensification (SRI). SRI- FAQS published by CIIFAD, USA.

Yadav, S., Gill, G., Humphreys, E., Kukal, S.S., Walia, U.S., 2011. Effect of water management on dry seeded and puddled transplanted rice. Part 1: Crop performance. Field Crops Research. 2011; 120(1), 112-122. Available from:sudhir.yadav@adelaide.edu.au.

Thorie, M., Sarkar, N.C., Kharutso, K., 2013. Effect of biofertilizer on the productivity of terraced upland rice (Oryza Sativa L.). International Journal of Bio-resource and Stress Management 4(3), 400-403. 